medicines chronically (7.4 medicines/patient). 563 discrepancies were detected in 170 patients (88.5\%): 372 discrepancies did not require clarification and 191 discrepancies required clarification with the physician. Among the discrepancies requiring clarification, $37.7 \%$ were accepted by the physician as reconciliation errors (REs). Most were due to the omission of the patient's chronic treatment. Most REs were associated with cardiovascular drugs, nervous system drugs and gastrointestinal drugs. The severity of RE was mostly classified within category C but $30.6 \%$ had category D and $4.2 \%$ had category E (potential harm).

Conclusions The reconciliation process has detected the existence of discrepancies in patients older than 75 years. Special attention should be paid to drugs belonging to the cardiovascular system, nervous system and the digestive system. Most REs would probably not have caused damage but more than $30 \%$ had category $\mathrm{D}$ and $\mathrm{E}$.

No conflict of interest.

\section{GRP-116 MEDICINES RECONCILIATION: AN INNOVATIVE COMPUTER-BASED USE OF THE MEDICINES LIST}

doi:10.1136/ejhpharm-2013-000276.116

'R Palmeira de Oliveira, 'S Morgado, ${ }^{2} \mathrm{P}$ Patrício, ${ }^{2} \mathrm{P}$ Riscado, 'MO Fonseca. ${ }^{1} \mathrm{Hospital}$ Center of Cova da Beira, Pharmacy, Covilhã, Portugal; ${ }^{2}$ Hospital Center of Cova da Beira, Information Technology, Covilhã, Portugal

Background Medicines reconciliation is as an important approach to prevent medicines errors and adverse health outcomes. However, the implementation of these interventions is frequently unsuccessful especially due to difficulties in information access and communication.

Purpose To analyse the outcomes of a computer programme developed to summarise patients' medicines on a list including additional information for the patient.

Materials and Methods Descriptive analysis was performed of medicines lists created from May 2010 to September 2012. The impact of the project was measured through a questionnaire on patients' opinions about the medicines list.

Results A computer programme was developed by our hospital multidisciplinary team. A database was created by adding to the National Medicines Database information written for patients, by pharmacists, on how to take some of the medicines and their therapeutic goals. Monthly updates are performed to include or eliminate medicines.

Access for physicians is available throughout the hospital for reconciliation at discharge and consultation, allowing for medicines lists updates.

Over the study period 1057 medicines lists were completed for 720 different patients. Neurology and Internal Medicine doctors were the most frequent users of this computer programme. Polypharmacy and individual motivation were identified as the main factors for physicians' adherence.

Specially-written information was available for $17 \%$ of the total database medicines by September 2012. Considering the lists, $55 \%$ of the medicines included this information since the most commonly-used therapeutic groups had been selected as high priority for information development by pharmacists.

A total of 48 patients and caregivers answered the questionnaire. $87 \%$ considered that the lists were very useful in medicines management at home while $92 \%$ thought that the written information was very clear.

Conclusions The programme we created is an effective tool for medicines reconciliation and is accepted by patients. This approach may improve patients' knowledge and medicines use at home, reducing medicines errors.

No conflict of interest.

\section{GRP-117 MEDICINES WITH ANTICHOLINERGIC ACTIVITY IN ELDERLY PATIENTS}

doi:10.1136/ejhpharm-2013-000276.117

${ }^{1} \mathrm{M}$ Hernandez, ${ }^{1 B}$ Llagostera, ${ }^{1} \mathrm{M}$ Espier, ${ }^{2} \mathrm{C}$ Minguell. 'Mutuam, Prescription Quality Unit, Barcelona, Spain; ${ }^{2}$ Mutuam, Geriatric care teams EAR director, Barcelona, Spain

Background Medicines with anticholinergic activity have been linked to a variety of adverse drug reactions in the elderly.

Purpose To determine the anticholinergic burden in revised profiles, and the level of risk.

Materials and Methods The Prescription Quality Unit (PQU), which is staffed by a doctor, two pharmacists, a nurse and other technical-administrative staff, is integrated into the geriatric care team. The Unit provides care to 6800 residents in 163 centres.

The PQU provides training and support to different care teams by reviewing procedures and holding conciliation meetings. The process of rationalisation consists of systematically reviewing medicines plans according to the criteria of efficacy, safety and efficiency. The team suggests modifications in medicines plans and reports to the health care professionals involved. Anticholinergic drugs were selected from the review. These medicines were classified into four groups, according to the anticholinergic potency.

Results A prospective study was undertaken during the period June 2011-June 2012: 7,347 patients were reviewed (some in duplicate). 959 patients were identified, and those patients were prescribed 1,984 drugs with anticholinergic activity (mean age 85 years (52-111 years)).

In 162 patients, strong anticholinergic activity drugs were found: $62 \%$ oral antimuscarinics for urinary incontinence, 33\% tricyclic antidepressants, $4 \%$ antispasmodics with anticholinergic properties and $1 \%$ systemic $\mathrm{H}_{1}$ antihistamines (dexchlorpheniramine); 252 patients with moderate anticholinergic activity drugs $(70 \%$ paroxetine); 500 patients with mild anticholinergic drugs and 45 patients had drugs whose activity was concentration-dependent.

Sixty-eight patients were simultaneously being prescribed more than one medicine with anticholinergic activity (17 patients on strong anticholinergic activity drugs simultaneously).

Conclusions Due to the comorbidities and frailty of this population, medicines must be selected individually for each patient, selecting drugs with the lowest level of anticholinergic activity. We observed a group of patients at special risk who were being treated for pathologies related to the urinary tract.

No conflict of interest.

\section{GRP-118 MONITORING PATIENTS TREATED WITH DRONEDARONE}

doi:10.1136/ejhpharm-2013-000276.118

$\mathrm{J}$ Alonso Herreros, DM Escudero Galindo, JM Bernal Montañes. Hospital Universitario Reina Sofia, Pharmacy, Murcia, Spain

Background Dronedarone is a drug related to amiodarone, marketed in 2010. Soon after, there were several safety alerts that forced Health Authorities to reduce their use, and require hepatic and renal function cheques. The alert (reference SGMUH (FV), 16/2011) requires initial and then at least 6-monthly hepatic and renal function tests.

Purpose To assess the degree of compliance with the analytical tests required by the Competent Authority in patients taking dronedarone (creatinine and liver enzymes) in a health area of 200,000 inhabitants.

Materials and Methods We selected patients who were prescribed dronedarone during the last half of 2011. These patients were identified by querying the electronic prescription billing system. The prescriptions were analysed in three groups of patients: those who started treatment after publication of the alert so cheques 